\title{
Gene expression patterns associated with p53 status in breast
}

\section{cancer}

\author{
Melissa A Troester*1, Jason I Herschkowitz ${ }^{2,3}$, Daniel S Oh²,3, Xiaping $\mathrm{He}^{2,4}$, \\ Katherine A Hoadley ${ }^{2,3}$, Claire S Barbier ${ }^{5}$ and Charles M Perou ${ }^{2,4,5}$
}

\begin{abstract}
Address: ${ }^{1}$ Division of Biostatistics and Epidemiology, School of Public Health and Health Sciences, University of Massachusetts Amherst, Amherst MA, USA, ${ }^{2}$ Lineberger Comprehensive Cancer Center, University of North Carolina at Chapel Hill, Chapel Hill, NC, USA, ${ }^{3}$ Curriculum in Genetics and Molecular Biology, University of North Carolina at Chapel Hill, Chapel Hill, NC, USA, ${ }^{4}$ Department of Genetics, University of North Carolina at Chapel Hill, Chapel Hill, NC, USA and ${ }^{5}$ Department of Pathology and Laboratory Medicine, University of North Carolina at Chapel Hill, Chapel Hill, NC, USA

Email: Melissa A Troester* - troester@schoolph.umass.edu; Jason I Herschkowitz - jih@unc.edu; Daniel S Oh - daniel_oh@med.unc.edu; Xiaping He - xiaping@med.unc.edu; Katherine A Hoadley - hoadley@med.unc.edu; Claire S Barbier - camus@med.unc.edu;

Charles M Perou - cperou@med.unc.edu

* Corresponding author
\end{abstract}

Published: 06 December 2006

BMC Cancer 2006, 6:276 doi:10.1 I86/147/-2407-6-276
Received: 30 October 2006

Accepted: 06 December 2006

This article is available from: http://www.biomedcentral.com/147/-2407/6/276

(C) 2006 Troester et al; licensee BioMed Central Ltd.

This is an Open Access article distributed under the terms of the Creative Commons Attribution License (http://creativecommons.org/licenses/by/2.0), which permits unrestricted use, distribution, and reproduction in any medium, provided the original work is properly cited.

\begin{abstract}
Background: Breast cancer subtypes identified in genomic studies have different underlying genetic defects. Mutations in the tumor suppressor p53 occur more frequently in estrogen receptor (ER) negative, basal-like and HER2-amplified tumors than in luminal, ER positive tumors. Thus, because $\mathrm{p} 53$ mutation status is tightly linked to other characteristics of prognostic importance, it is difficult to identify $\mathrm{p} 53$ 's independent prognostic effects. The relation between $\mathrm{p} 53$ status and subtype can be better studied by combining data from primary tumors with data from isogenic cell line pairs (with and without p53 function).

Methods: The p53-dependent gene expression signatures of four cell lines (MCF-7, ZR-75-I, and two immortalized human mammary epithelial cell lines) were identified by comparing p53-RNAi transduced cell lines to their parent cell lines. Cell lines were treated with vehicle only or doxorubicin to identify p 53 responses in both non-induced and induced states. The cell line signatures were compared with $\mathrm{p} 53$-mutation associated genes in breast tumors.

Results: Each cell line displayed distinct patterns of p53-dependent gene expression, but cell type specific (basal vs. luminal) commonalities were evident. Further, a common gene expression signature associated with $\mathrm{p} 53$ loss across all four cell lines was identified. This signature showed overlap with the signature of p53 loss/mutation status in primary breast tumors. Moreover, the common cell-line tumor signature excluded genes that were breast cancer subtype-associated, but not downstream of $\mathrm{p} 53$. To validate the biological relevance of the common signature, we demonstrated that this gene set predicted relapse-free, disease-specific, and overall survival in independent test data.

Conclusion: In the presence of breast cancer heterogeneity, experimental and biologically-based methods for assessing gene expression in relation to p53 status provide prognostic and biologically-relevant gene lists. Our biologically-based refinements excluded genes that were associated with subtype but not downstream of $\mathrm{p} 53$ signaling, and identified a signature for $\mathrm{p} 53$ loss that is shared across breast cancer subtypes.
\end{abstract}




\section{Background}

The tumor suppressor p53 is mutated in $30 \%$ of breast cancers [1], but rates of p53 mutation vary depending upon the subtype of breast cancer. For example, p53 mutations are found more frequently in aggressive estrogen receptor (ER)-negative breast cancers [1], and have been shown to correlate with breast cancer subtype in gene expression studies [2] and in a population-based study [3]. Genetic abnormalities such as amplified HER2/ ERBB2 [1] and aneuploidy [4] are also frequently associated with p53 mutation status. These correlations suggest intrinsic heterogeneity of p53 signaling across breast cancer subtypes.

Gene expression studies can help to characterize breast cancer heterogeneity. Previous in vitro studies of gene expression have demonstrated that cell line models of luminal breast cancers show a strong stress response following chemotherapeutic treatment, with notable changes in p53-regulated genes such as p21 (Cip1). The same magnitude of p53-regulated responses was not observed for cell line models of basal-like breast cancer [5]. Inherent differences in p53 signaling and function according to cell type of origin could account for the association between rates of p53 mutation and breast cancer subtype. In this study, we engineered isogenic cell line pairs with and without p53 function using RNA interference (RNAi) and examined the stress responses of parent and RNAi-transduced cell lines. Our aim was to assess how variation in cell line backgrounds alters the effects of p53 loss. We also aimed to identify a common response to p53 loss that is shared by most breast cancers. Thus, we compared the lists of p53-responsive genes in vitro to gene lists derived from in vivo breast tumor data to identify a set of common p53 responsive genes. The biological relevance of this common p53 signature was assessed by using this gene list to predict outcomes on independent test data sets of breast cancer patients.

\section{Methods}

\section{Cells and culture conditions}

Two hTERT immortalized Human Mammary Epithelial (HME) cell lines (ME16C and HME-CC) and two established breast cancer cell lines (MCF-7 and ZR-75-1) were cultured as described previously [5]. A mitochondrial dye conversion (MTT) assay was used to measure cell line responses to $36 \mathrm{~h}$ of treatment with $0-10 \mu \mathrm{M}$ doxorubicin hydrochloride (DOX) [5].

Short hairpin RNAs (RNAi) against p53 were constructed using a 19-mer sequence (GACTCCAGTGGTAATCTAC) described previously [6], but using the pSUPER.RETRO.puro vector with stuffer (Oligo Engine, Seattle, WA). A version of this vector containing two mismatches within the 19-mer sequence (GACTCCGGTT-
GTAATCTAC) was also prepared as a mismatch control. HEK-293T cells were transfected with 10 micrograms each of pSUPER.RETRO.puro vector, pVpack-GP (Stratagene), and pVpack-Ampho (Stratagene) using Lipofectamine Reagent and PLUS Reagent (Invitrogen). Supernatants containing replication-incompetent retrovirus were collected 48 hours after transfection and applied to all four cell lines. Stable populations of cell lines expressing p53RNAi or mismatch-RNAi were selected for two weeks in 1 $\mu \mathrm{g}$ per $\mathrm{mL}$ puromycin.

\section{Western blots}

Cells were treated for $24 \mathrm{~h}$ with $1 \mu \mathrm{M}$ DOX, and cell free extracts, protein quantitation, and denaturation were as described previously [5]. Forty $\mu \mathrm{g}$ of protein were electrophoresed on a $4-20 \%$ Tris-HCl Criterion precast gel (BioRad) and transferred to a Hybond-P membrane (Amersham Biosciences) by electroblotting. The blots were probed with antibodies against p53 (Santa Cruz; D01) and $\beta$-actin (Abcam, AC-15) and then with antimouse IgG horseradish peroxidase linked whole antibody from sheep (Amersham). Enhanced chemiluminescence was used for detection (SuperSignal West Pico Chemiluminescent Substrate, Pierce).

\section{Microarray experiments}

Cell lines were grown, treated for 12,24 , or $36 \mathrm{~h}$ with DOX at the IC50 concentration, and harvested using a previously described protocol [5]. Feeding control (sham) and reference mRNA samples were prepared as described previously [5]. Cy3- and Cy5-labeled cDNAs were synthesized from control or treated cell line mRNA, respectively, according to a direct labeling protocol (Agilent Technologies), and were hybridized to Human 1A oligonucleotide arrays (Agilent Technologies). All microarray raw data tables have been deposited in the Gene Expression Omnibus under the accession number of GSE3178 (submitter C. Perou).

\section{Identification of p53-dependent DOX-response signature from microarray data}

For all comparisons, in vitro and in vivo as described below, genes that were significantly different in expression were identified using a 2-class, unpaired Significance Analysis of Microarrays (SAM) [7]; for the SAM analysis, the data were first filtered to exclude genes that did not have mean signal intensity greater than twice the median background value for both the red and green channel in at least $70 \%$ of the experiments. The SAM delta values were adjusted to obtain the largest gene list that gave a false discovery rate of less than 5\%. Using the SAM-derived gene lists, average linkage hierarchical cluster analysis was conducted using Pearson correlation in the Cluster program and the data were visualized in Treeview $[8,9]$. EASE, the 
Expression Analysis Systematic Explorer was used to identify enriched biological themes in gene lists [10].

Each cell line was examined for p53 response in both untreated and DOX-treated states. To identify the gene expression effects of p53 loss in DOX-treated cells (p53induced state), parent cell lines treated with $\operatorname{DOX}(\mathrm{n}=3$ for each cell line) were compared to RNAi-transductants treated with DOX ( $\mathrm{n}=3$ for each cell line). To identify the gene expression effects of p53 loss in the absence of DOX treatment, sham-treated parent cell lines (wildtype p53) were compared to sham-treated RNAi-transductants $(\mathrm{n}=$ 3 for both treatment groups in each cell line). However, to derive a list of genes that were differentially expressed in both in vitro and the in vivo data sets, the common p53 response across all four cell lines was the most relevant. Thus, we also performed an analysis comparing all RNAitransduced cell line experiments $(n=24)$ to all parent cell line experiments $(n=24)$. The resulting list represented the common response to p53 loss across cell lines.

To identify the gene expression signature associated with p53 in vivo, we used primary breast tumor data $[2,11,12]$ that is publicly available from the Stanford Microarray Database and the Gene Expression Omnibus. DOXtreated patients for which p53 status had been determined by sequence analysis [2] were included in our analysis (102 tumor samples, including 8 normal-like breast samples, one unclassified tumor, and 37 before and after pairs, representing 69 patients in total). All tumor subtypes described in Sorlie et al. [2] [classified using intrinsic analysis [12]] were included, except true normal breast and normal-like breast tumor samples. This sample set also included tumors collected before and after treatment with doxorubicin. The gene expression patterns of the p53 mutant samples $(n=43)$ were compared to those of the p53 wildtype samples $(n=52)$.

\section{Identification of p53 functional status in independent test data sets}

A final 52 gene list was derived by identifying those genes that were differentially expressed in response to p53 loss in both the in vitro and in vivo data sets. These genes were matched to publicly available array data $[13,14]$, using unique Unigene identifiers. Of the 52 genes, 48 and 50 were present on the Chang et al. data set and Miller et al. data sets, respectively. Microarray platform/source systematic biases between the training and the test sets were corrected using Distance Weighted Discrimination (DWD) [15]. To classify tumors in the independent test sets (Chang et al. or Miller et al.) as p53-functional or not, two centroids were created using the Sorlie et al. training set. The centroids were based on average gene expression in tumors in Figure 5A (mutant enriched) vs. that of tumors in Figure 5B (wildtype enriched). Each Chang et al. or Miller et al. tumor was classified according to the nearest centroid as determined by Spearman correlation.

\section{Other statistical analyses}

Survival analyses were conducted using Sorlie et al. tumor data (excluding duplicate samples from the same person, resulting in a total of 66 patients representing 31 diseasespecific and 26 overall survival events for survival analyses), Change et al. tumor data [337 patients: 295 patients from [13] and 42 tumors published in an earlier paper [16] from the same group, representing 126 disease specific and 79 overall survival events] and Miller et al. [14] data (236 patients, 52 disease specific events). For analyses of the Miller et al. dataset, patients that had survived at least ten years were censored to be consistent with previous analyses [14]. Kaplan Meier analyses were conducted using WinStat for Microsoft Excel.

Because the large data set of Chang et al. also included data on other prognostic variables, Cox proportional hazards modeling was conducted (SAS version 9.1). The reduced model that included ER status (positive vs. negative), tumor size ( $\leq 2 \mathrm{~cm}$ vs. $>2 \mathrm{~cm}$ ), lymph node status (indicator coding with three categories: $0,1-3,>3$ positive nodes or metastatic), age (in decades), grade (indicator coding with three categories: 1, 2, 3), and treatment (yes if treatment with chemo and/or hormonal therapy, no if no adjuvant therapy) was compared to a full model that also included a binary variable indicating p53 classification (based on gene-expression).

To determine if p53 status differed according to tumor subtype, a Fisher-Freeman-Halton (FFH) exact test was conducted using SAS version 9.1 (Cary, NC). Analyses of sequence-based mutation characteristics (e.g. missense/ in-frame vs. nonsense and frameshift, missense DNA binding vs. non-DNA binding) in association with gene expression classification were also conducted using FFH exact tests.

\section{Results}

Gene expression and phenotypic analysis of cell lines expressing p53 RNAi

To study the effects of p53 loss in vitro, an RNAi construct specific for p53 [6] was stably expressed in MCF-7, ZR-751, ME16C and HME-CC cells. All four cell lines had wildtype p53 sequence and expressed functional p53 (showed p53 induction in response to treatment with DOX, Figure 1) prior to transduction with the p53-RNAi retroviral construct. Expression of p53-RNAi substantially knocked down p53 protein levels in both treated and untreated cells (Figure 1).

The phenotypic effects of p53 knock-down varied by cell line (Figure 2). MCF-7 cells became more resistant to 


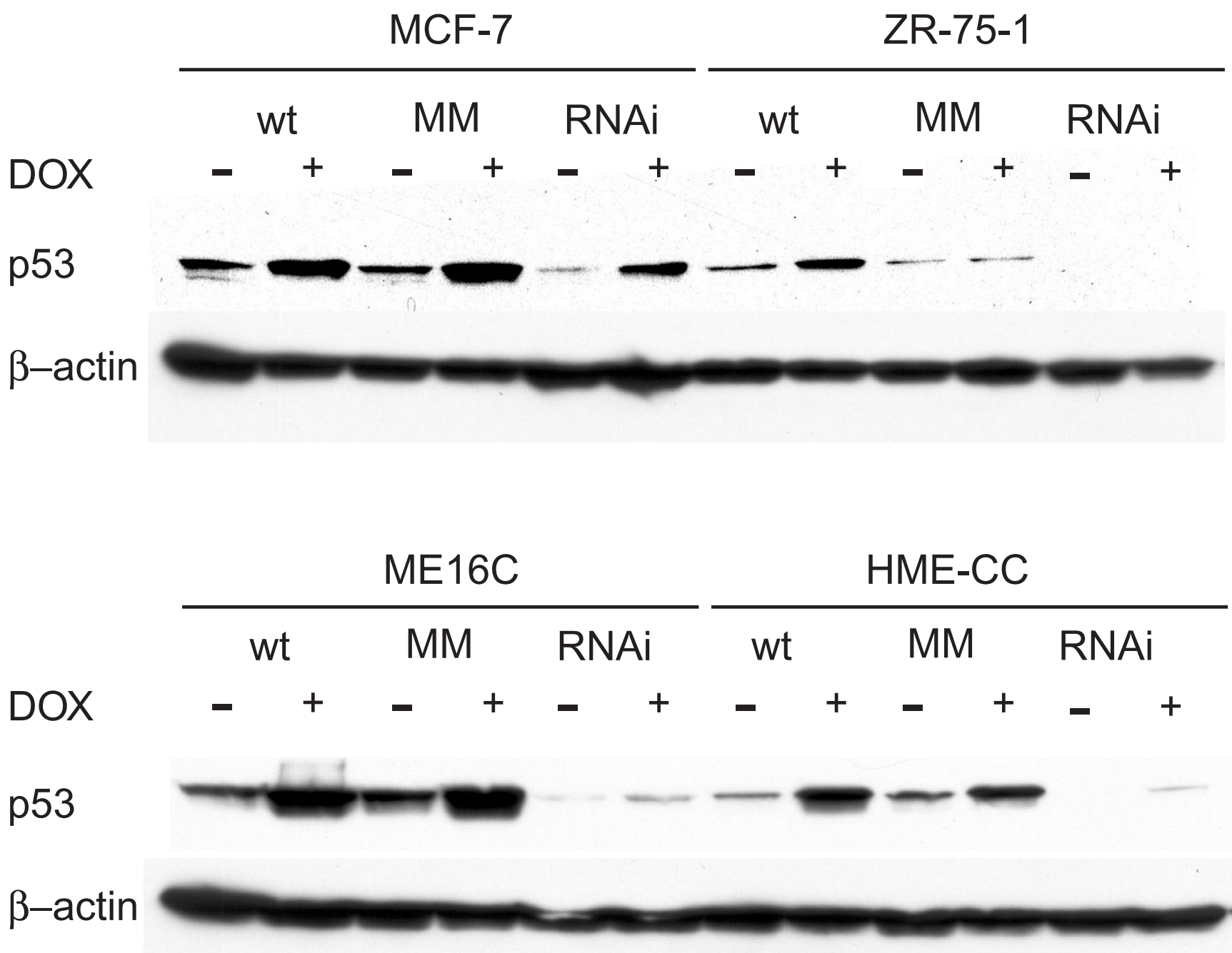

Figure I

p53 protein expression is knocked down by RNAi expression. The expression of p53 was examined by Western blot analysis of extracts from MCF-7, ZR-75-I, HME-CC, and MEI6C cell line parents and the same cell lines stably transduced with p53-targeted RNAi vector or p53 mismatch (MM) RNAi vector. Treatment with doxorubicin $(24 \mathrm{~h}, \mathrm{I} \mu \mathrm{M})$ induced p53 expression in all cell lines and transductants, but induced levels were markedly lower in the p53-RNAi cells.

DOX, while ZR-75-1, ME16C and HME-CC cells displayed no change in DOX sensitivity. Consistent with the different responses in the DOX sensitivity assay, gene expression signatures significantly associated with p53 loss (in 2-class SAM analyses) were different for each cell line and cell type (gene lists are given in Additional File 1). As shown in Figure 3, MCF-7 and ZR-75-1 cells showed a stronger p53-dependent signature following treatment with DOX. The immortalized HMECs, conversely, showed stronger p53-dependent signatures in the absence of DOX (i.e. parents vs. RNAi, both untreated). Analysis of SAMderived gene lists using gene ontology software (EASE) showed enrichment for categories of genes with known relevance to p53 function. For example, among the DOX- treated samples (DOX-treated parent vs. DOX-treated RNAi-expressing), three cell lines (HME-CC, MCF-7 and ZR-75-1) increased genes involved in mitosis after transduction with p53-RNAi. ME16C did not induce categories of mitosis genes, but did suppress negative regulators of cell proliferation. Significant down-regulation of apoptotic genes was only seen in ZR-75-1 cells.

The p53-response observed among DOX-treated cell lines differed from the p53-response in sham-treated cell lines. For example, the luminal-like cell lines (MCF-7 and ZR75-1) that had the largest transcriptional response to DOX, showed a modest response to p53 loss in shamtreated samples (sham-treated parent versus sham-treated 

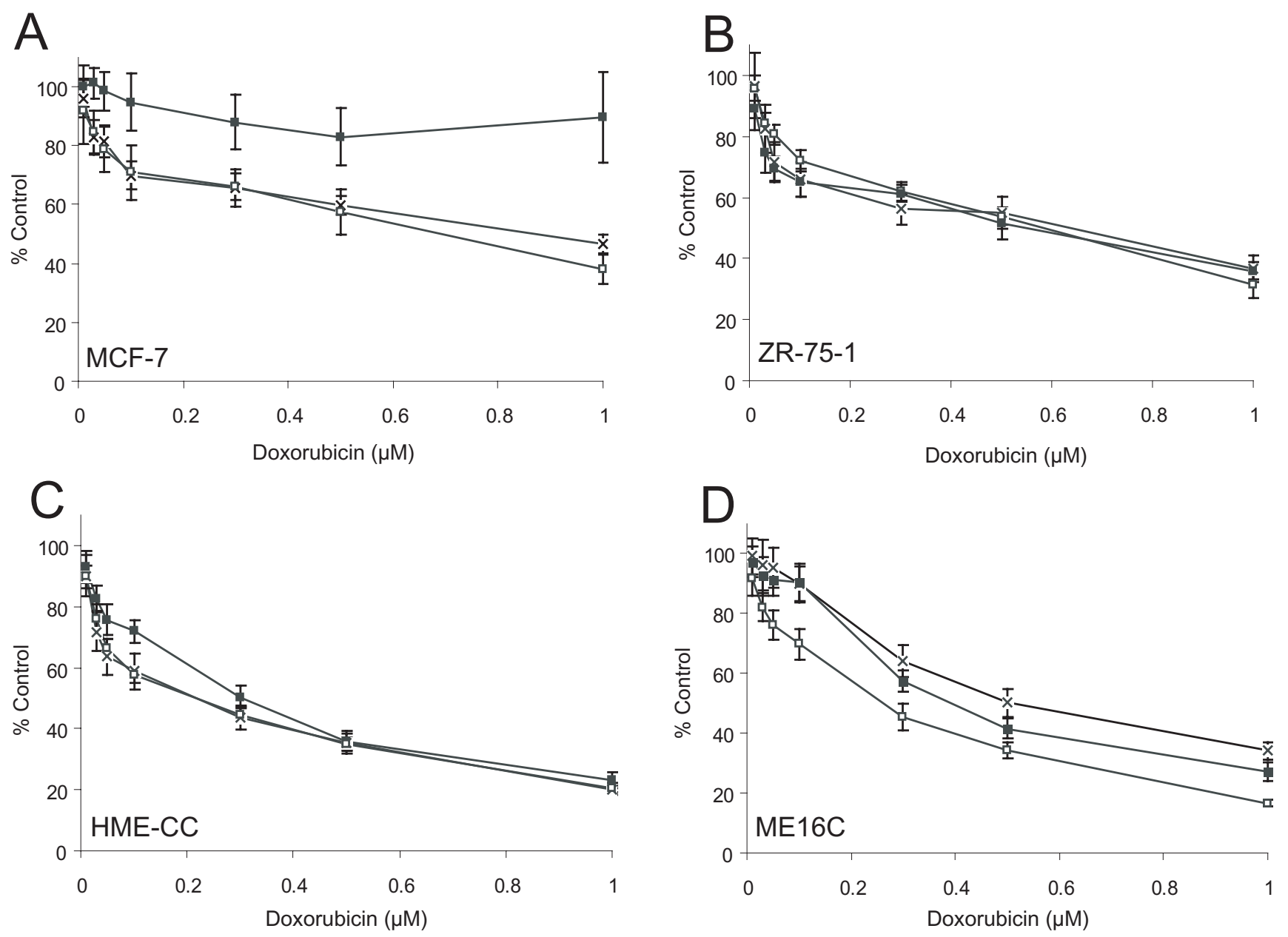

\section{Figure 2}

Chemosensitivity is altered in the MCF-7 cell line following transduction with p53-RNAi. MEI6C, HME-CC, and ZR-75-I cell lines had similar chemosensitivity curves for wildtype (x), p53-targeted RNAi expressing cells (solid square) and p53 mismatch RNAi expressing cells (open square). Only the p53-RNAi MCF-7 cells showed significant chemoresistance.

RNAi-expressing). Sham-treated MCF-7 cells showed no significant changes and ZR-75-1 cells showed few changes in response to p53 loss. EASE analysis of the ZR-75-1 changes did not identify categories with clear relevance to p53 signaling. Only one down-regulated gene ontology category (extracellular region) was identified. Induced gene categories were transition metal homeostasis genes and genes with unknown roles in biological processes. However, among the basal-like cell line models, ME16C significantly down-regulated anti-apoptosis genes and HME-CC significantly up-regulated mitosis/proliferation genes. The strong mitotic signature of sham-treated HMECC cells showed overlap with the strong mitotic signature observed in DOX-treated HME-CCs. Thus, p53 loss had different effects across cell type and cell line.

Common patterns of expression shared by most of the four lines were identified using a 2-class SAM (DOX- and sham-treated combined from all parental lines vs. all p53RNAi expressing lines) to analyze all four cell lines simultaneously. In addition to identifying a common response, this analysis had a larger sample size and thus, had better power to detect a broader range of p53-regulated genes. There were 696 genes which responded significantly to p53 loss in the cell lines (1). Included in this list were many known direct p53 targets including MDM2, p21 (Cip1), GADD45A, and ribonucleotide reductase M2. All of these genes had lower expression in p53-RNAi lines, consistent with expectation. In total, 357 of the 696 significantly altered genes had lower expression in p53-RNAi lines; EASE analysis indicated that apoptosis genes, cell death genes, and regulators of programmed cell death were significantly over-represented. Conversely, there were 339 genes (of 696 significantly altered genes) that were more highly expressed in RNAi lines, including genes 


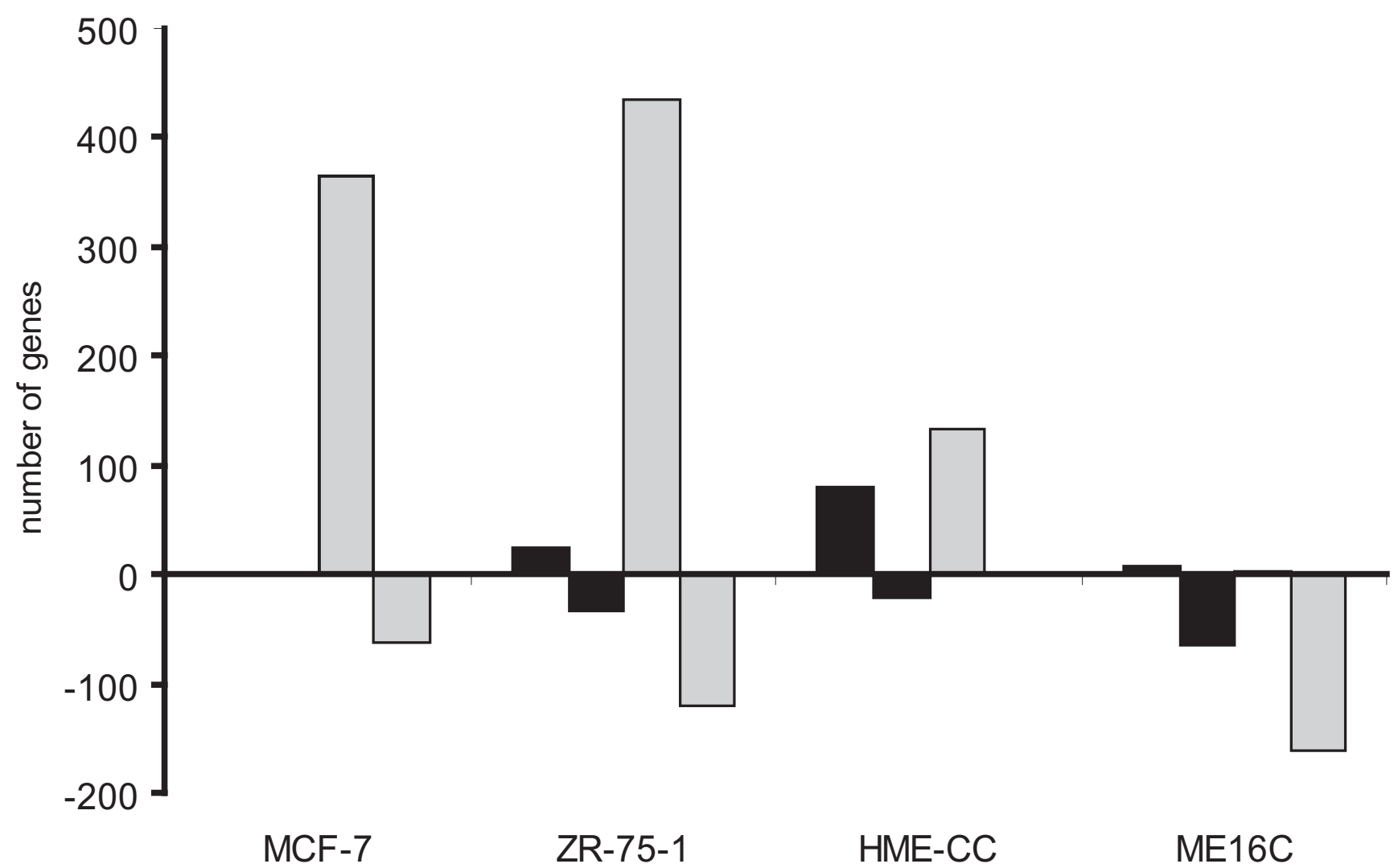

Figure 3

Number of genes with significantly different expression following p53 loss (by RNAi) in sham-treated (black) or doxorubicin-treated (gray) cells. Sham-treated cells represented feeding controls, treated with fresh media and harvested at the same time points as the doxorubicin treated cells. Positive $y$-axis indicates number of genes up-regulated and negative $y$ axis indicates number of genes down-regulated by $\mathrm{p} 53$ knockdown. Luminal cell lines had the largest transcriptional response to $\mathrm{p} 53$ loss following induction, while HMEC cell lines had a stronger signature for p53 loss in the uninduced state.

involved in mitosis, cell cycle control, and regulation of DNA repair.

\section{Gene expression signatures of primary tumors with wild- type and mutant $\mathbf{p 5 3}$}

Gene expression data for primary breast tumors with known p53 mutation status is publicly available [2,12]. Using this data, we found that the expression of 747 genes was significantly correlated with p53 status (Figure 4A). The hierarchical cluster of these genes across the primary tumors contained two branches (Figure 4B), one enriched for wild-type tumors (left branch, 45 of 53 wildtype samples) and one enriched for mutant tumors (right branch, 34 of 42 mutant samples). A proliferation cluster/signature was differentially expressed across the two branches of the dendrogram (Figure 4C). This cluster had higher expression in p53 mutants, and included the cell cycle associated genes cyclin A2, CDC28 subunit 1B, CDC2, cyclin-dependent kinase inhibitor 3, polo-like kinase, and topoisomerase IIA. EASE analysis confirmed that genes involved in mitosis and cell cycle progression were significantly over-represented in the set of genes that had higher expression in p53 mutant tumors.

A cluster (Figure 4D) enriched for genes associated with the luminal/ER+ tumor subtypes (N-acetyltransferase 1, estrogen receptor 1 , putative G-protein-coupled receptor, trefoil factor 3, GATA binding protein 3, and X-box binding protein 1) was also present in this gene set $[2,11,12]$. This cluster was more highly expressed in wildtype tumors, likely due to a larger representation of luminal tumors in this branch. In fact, when the intrinsic subtype of each of the patients in Figure 4 was determined by clustering all 95 tumor samples using the intrinsic list of Sorlie et al. [12], a statistically significant association between p53 status and tumor subtype was observed ( $p=0.002)$, with $31 \%$ of luminal tumors and $80 \%$ of basal-like tumors having mutant p53. Because the frequencies of 

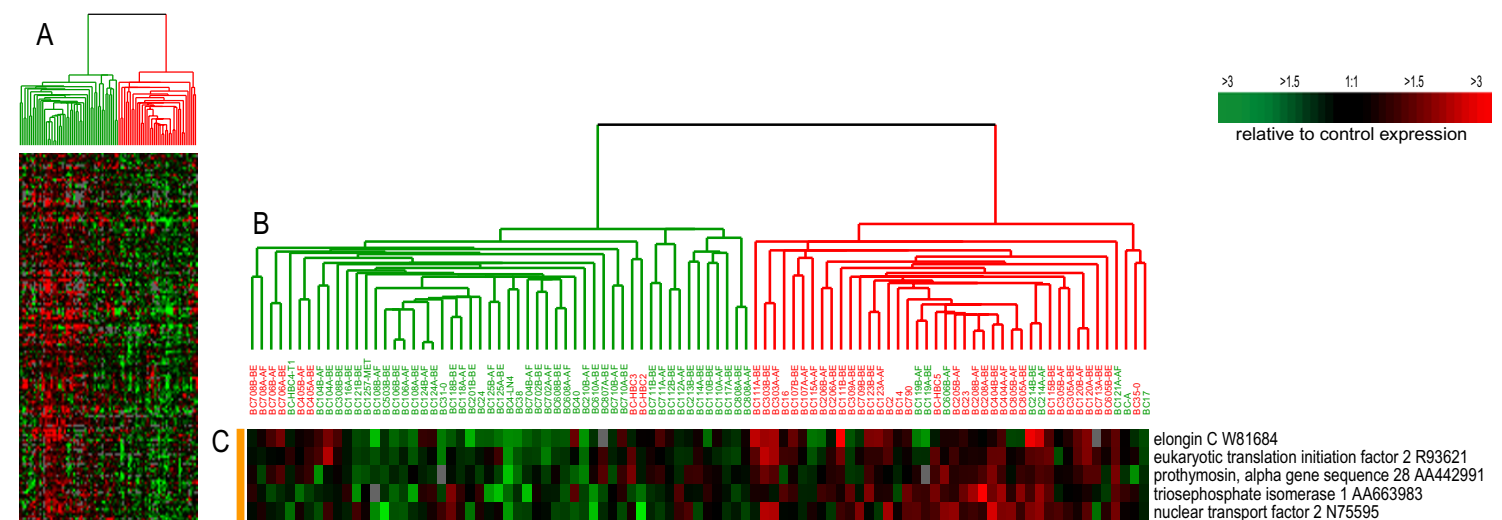

elongin C W81684
eukaryotic translation initiation factor 2 R93621
prothymosin, alpha gene sequence 28 AA442991 prothymosin, alpha gene sequence 28 AAA42
triosephosphate isomerase 1 AA663983 nuclear transport factor 2 N75595 chromosome 10 open reading frame 7 AA448289 small nuclear ribonucleoprotein polypeptide C AA253448 HSPC163 protein AA05313 CNAP1 AA668256 high mobility group AT-hook 1 AA448261 phosphofructokinase, platelet AA608558 BTG family, member 3 N52496 gamma-glutamyl hydrolase AA455800
neuregulin 1 R72075 neuregulin 1 R72075
MCM4 AA485983 barren homolog Drosophila N54344 AA026682
v-myb2 AA456878 v-myb2 AA456878
polymyositis/scleroderma autoantigen 1 AA458994 polymyositis/scleroderma autoantigen 1 AA458994
forkhead box M1 AA129552 CDC28 protein kinase regulatory subunit $1 B$ AA459292 E2F transcription factor $1 \mathrm{H} 61303$ cyclin-dependent kinase inhibitor 3 AA284072 ubiquitin-conjugating enzyme E2C AA430504 mitogen-activated protein kinase 13 AA15749 trophinin associated protein tastin H94949
karyopherin alpha 2 RAG cohort 1 AA676460 ubiquitin carrier protein AA464019 pituitary tumor-transforming 1 AA430032 CDC28 protein kinase regulatory subunit 2 AA292964 CDC28 protein kinase regulatory subunit 2 AA010065
CDC20 cell division cycle 20 homolog AA598776 CDC20 cell division cycle 20 homolog AA598776
chromosome 10 open reading frame 3 AA131908 cell division cycle 2, G1 to S and G2 to M AA598974 cyclin A2 AA608568 kinesin family member 23 AA452513 centromere protein F, 350/400ka mitosin AA701455 polo-like kinase Drosophila AA629262
thyroid hormone receptor interactor 13 AA630784 MAD2 mitotic arrest deficient-like 1 yeast AA48107 serine/threonine kinase 6 R11407 topoisomerase DNA II alpha $170 \mathrm{kDa}$ AA504348 replication factor C activator 1 4, 37kDa H54751 hematological and aeurological expressed 1 AA45986 tubulin, alpha 1 testis specific AA180742

AA010188
PRO2000 protein $\mathrm{H} 58234$ PRO2000 protein H58234
kinesin family member C1

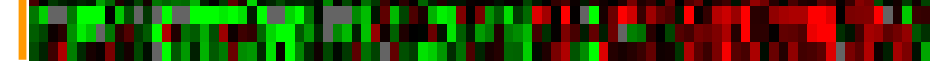
esin family member $\mathrm{C} 1 \mathrm{~N} 69491$

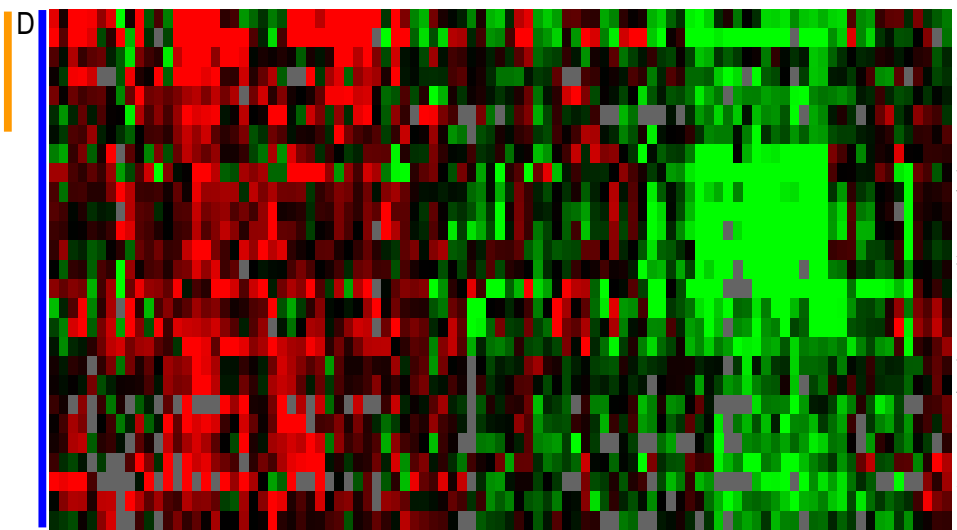

$\mathrm{N}$-acetyltransferase 1 R91802
$\mathrm{N}$-acetyltransferase 1 T67128 $\mathrm{N}$-acylsphingosine amidohydrolase 1 AA664155 estrogen receptor 1 AA164585 hypothetical protein DKFZp434L142 N95180 methylcrotonoyl-Coenzyme A carboxylase 2 beta N31952 hydroxysteroid 17-beta dehydrogenase 4 AA4
putative G protein-coupled receptor $\mathrm{H} 50224$ putative $G$ protein-coupled recep
trefoil factor 3 intestinal N74131 fms-related tyrosine kinase 1 AA058828 GATA binding protein 3 R31441 $X$-box binding protein 1 W9012 T74639

estrogen receptor 1 AA291702 nuclear receptor binding protein W79308 hypothetical protein LOC255743 AA029948 T55728

hypothetical protein FLJ21062 AA02817 chromosome 14 open reading frame 45 AA460298 KIAA0876 protein AA431721
hypothetical protein FLJ10980 N45467 signal peptide, CUB domain, EGF-like 2 W74079 hypothetical protein FLJ14015

Figure 4

Gene expression pattern of tumor samples for 747 genes correlated with p53 status in tumors. The fold change relative to the median expression value across all tumors is shown. Colored bars in $A$ illustrate the location of clusters $C$ and $D$. The dendrogram in B shows that experiments were divided into two primary branches, one enriched for mutant (red) and one enriched for wildtype (green) tumors. Tumor sample names are red for mutants and green for wildtypes based on sequence analysis. Clusters enriched for proliferation and cell cycle genes (C) and for luminal/estrogen responsive tumor markers (D) are shown. 

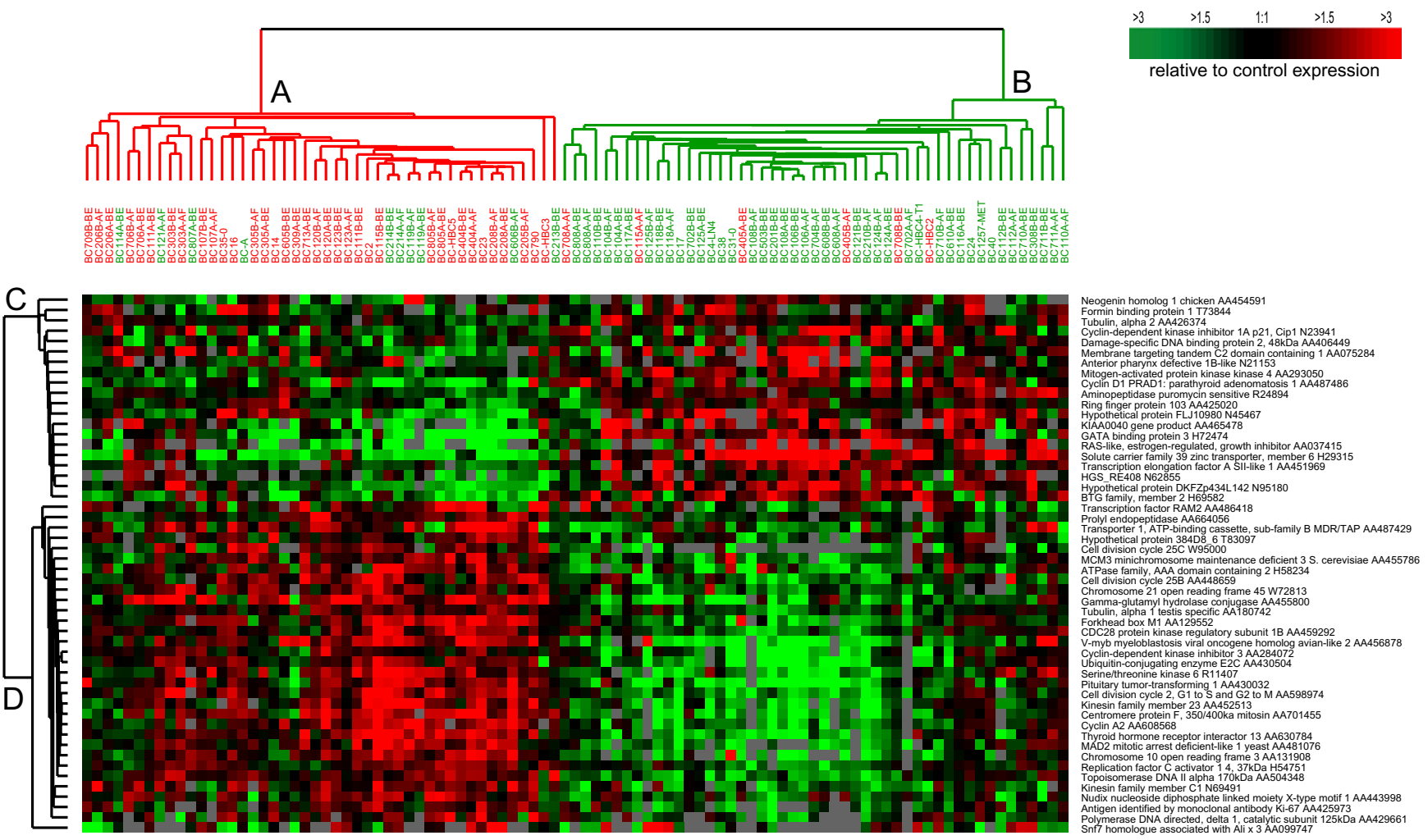

Figure 5

Cluster analysis of the tumor samples using $\mathbf{5 2}$ genes correlated with p53 status in tumors and cell lines. The fold change relative to the median expression value across all tumors is shown. The red dendrogram branch ( $A$ ) is enriched for p53 mutant tumors (sample names labeled red) while the green dendrogram branch (B) is enriched for p53 wild-type tumors (sample names labeled green). The dendrogram showing the gene clusters is shown in C and D.

p53 status varied significantly by subtype, the list of p53associated genes defined by SAM includes genes that were associated with subtype. Some of these genes may have no causal association with p53 defects, and thus, refinement of this list using our in vitro data was performed.

\section{Combined in vitro and in vivo analysis to identify p53- regulated genes}

The in vitro experiments that we conducted contained isogenic pairs of cell lines that were representative of both luminal and basal-like tumors. The in vivo experiments represented tumors derived from 69 different individuals, also representing both luminal and basal-like tumors. By comparing the p53-associated gene lists from the tumors to the cell lines, we refined our gene list and obtained a list of genes that were common to both data sets, representing a stereotypic p53 signature that held across diverse genetic backgrounds. There were 52 genes that were identified in common between the in vivo (747 genes) and in vitro (696 genes) lists. This 52-gene list retained GATA binding protein 3 and many of the proliferation cluster genes in Figure 4C (ATPase Family AAA domain containing 2, gamma-glutamyl hydrolase, MYBL2, CDC28 subunit 1B,
CDC2, cyclin A1). However, this list excluded ER and many of the luminal tumor-associated genes shown in Figure 4D. This list still contains a few p53-regulated genes that are also ER associated (such as GATA3), however their presence on this list cannot be viewed as an artifact of their association with ER status.

Patterns of expression for these 52 genes are shown across the primary tumor data in Figure 5. Again, two dendrogram branches were evident: one enriched for p53 mutants (Figure 5A) and the other enriched for p53wildtypes (Figure 5B). Figure 5 also shows two main clusters of genes, one of which (Figure 5C) was enriched for genes that are known to be p53-regulated including p21 (Cip1), BTG2, and damage-specific DNA binding protein 2. EASE analysis confirmed that this cluster, which had lower expression in mutant tumors, contained DNA damage response genes and negative regulators of cell proliferation. The second gene cluster (Figure 5D) was more highly expressed in mutant tumors, and EASE analysis confirmed that this cluster of genes was enriched for mitosis and proliferation genes. 


\section{Survival analyses using the 52-gene p53 signature} Kaplan-Meier survival analysis yielded highly significant survival differences between groups from Figure 5A (mutant-like) and 5B (wildtype-like) using the Sorlie et al. data. As shown in Figure 6, the 52-gene expression signature $(\mathrm{p}=0.001)$ significantly predicted overall survival (OS), while true mutation status on this set of samples was not significant $(\mathrm{p}=0.06)$. The expression signature $(\mathrm{p}$ $\left.=2.2 \times 10^{-5}\right)$ and true mutation status $(\mathrm{p}=0.001)$ also significantly predicted relapse-free survival (RFS). To further evaluate the prognostic value of this 52-gene signature, we performed survival analyses using two independent breast tumor data sets [published by Chang et al. (2005) and Miller et al. (2005)]. Kaplan-Meier analysis showed that this signature significantly predicted OS (Figure $5 \mathrm{C}, \mathrm{p}=$ $\left.7.2 \times 10^{-10}\right)$ and RFS $\left(\mathrm{p}=2.6 \times 10^{-7}\right)$ for the Chang et al. dataset, and disease-specific survival ( $\mathrm{p}=0.007$, RFS and OS data unavailable) for the Miller et al. dataset. We also performed multivariate analysis, using the Chang et al. dataset. Controlling for standard clinical predictors (ER, grade, node status, size, age, and treatment), Cox proportional hazards ratios were estimated for both OS $(\mathrm{RH}$, 95\% CI: 2.4, 1.3 - 4.4) and RFS (RH, 95\% CI: 1.9, 1.2 3.0). Thus, independent of standard clinical predictors the p53 expression classifier significantly predicted both OS $(\mathrm{p}=0.006)$ and RFS $(\mathrm{p}=0.006)$.

In our training data set (Sorlie et al.), the gene expression classifier had $82 \%$ agreement with sequence-based mutation status. True mutation status data was not available for the Chang et al. data set, but our classifier had $82 \%$ agreement with sequence-based mutation status in the Miller $e t$ al. data set. We were able to examine the location and type of mutations and compare them to classifier results using the Miller et al. data. Of the 29 mutants incorrectly classified as wildtype, 25 (86\%) were either missense mutations or in-frame insertions/deletions. This differs significantly ( $p=0.02$ ) from the percentage of mutations that were missense or in-frame among correctly classified mutants (58\%). Among the missense tumors, mutations in DNA binding domains of the $\mathrm{p} 53$ protein were also significantly more frequent $(\mathrm{p}=0.01)$ in tumors classified as mutant $(87 \%)$ than wildtype $(45 \%)$.

\section{Discussion}

Identification of a p53-responsive signature in breast cancer is confounded by associations with important tumor characteristics like ER status. The common p53 expression signature shared by cell lines and tumors in this study addressed this confounding by conducting cell lines experiments with ER positive and ER negative cell lines, and using experimental data to refine the gene lists derived from observational studies in patients. The resulting 52 gene, p53-associated list contained two biologically relevant gene clusters corresponding to downregulated and upregulated genes. This finding is consistent with the previous literature showing that p53 transactivates genes such as p21 and GADD45 and transrepresses genes such as topoisomerase IIA and CDC2. Inactivation of p53 affects both transactivation and transrepression to alter cell growth. Inactivation of p53 is also likely to cause downstream, indirect effects. As more research is conducted to identify pathway signatures $[17,18]$, evidence is growing that most, if not all, pathway signatures include both direct and indirect targets. However, these signatures still appear to show pathway-specific activity and represent valuable assays for pathway activity [19]. So, while we cannot conclude that these are exclusively direct targets of p53, genes in our signature do represent a common response to p53 loss in the breast.

This common p53-response list is biologically relevant, as shown by its ability to predict survival in patients across multiple true test data sets. Some of the genes in the common expression profile have been previously identified in other signatures of prognostic relevance (e.g. proliferation-associated genes) and are likely to be regulated by multiple oncogenic pathways. Our aim was not to identify a new prognostic signature that improves on previously published signatures. Rather, we aimed to demonstrate that events that are downstream of functional p53 loss are clearly associated with prognostic outcome, and are therefore biologically relevant. The predictive accuracy of p53dependent gene expression profiles [14] supports a role for p53 in breast cancer prognosis. Previous estimates of the relative hazard ( $\mathrm{RH})$ associated with p53 loss range from 1 (no effect) to 23 [20]. Our data suggests that this variability may relate to limitations of the methods for characterizing p53 status. p53 mutation status is most commonly characterized by direct DNA sequencing or by immunohistochemistry (IHC). Sequencing analysis cannot distinguish sequence variants with and without functional consequences. A meta-analysis of p53 mutation databases has demonstrated methodological biases associated with sequence-based mutation status [21]. IHC analysis treats accumulation of $\mathrm{p} 53$ protein as indicative of mutation; thus, IHC is biased toward identification of missense mutants and completely misses mutations that cause loss of p53 protein. With either IHC or sequence analysis, a narrow emphasis on p53 mutations can miss functional impairments in the p53 pathway (e.g. MDM2 amplification). These challenges could account for widely divergent estimates of p53's role in prognosis.

Our data analysis also showed that there was good agreement between mutation status and expression profiles. Using our 52 gene list, there was $>80 \%$ agreement between p53 mutation status and p53 expression class in both the Sorlie et al. and Miller et al. datasets. This high level of agreement across data sets attests to the fact that 

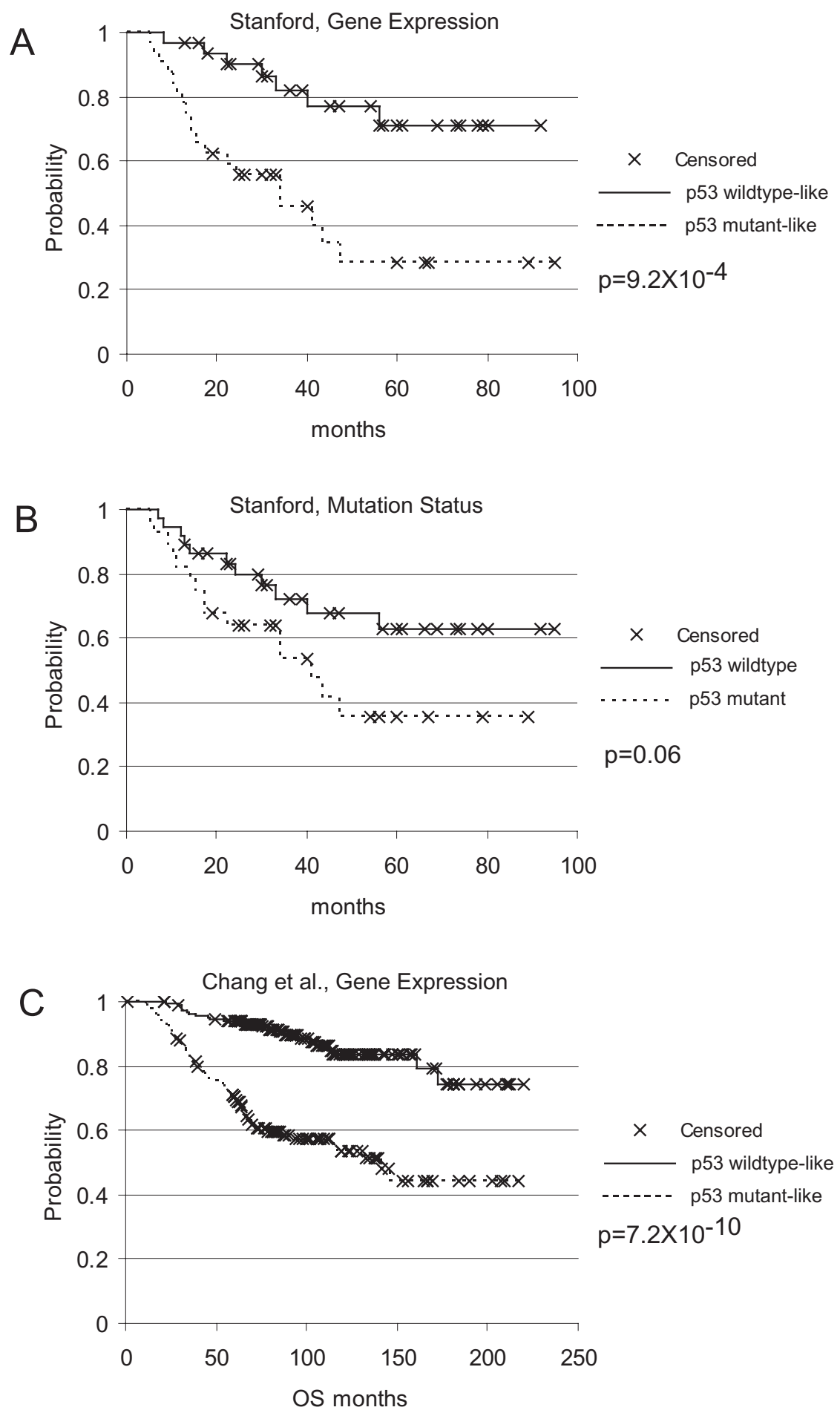

Figure 6

Kaplan-Meier survival curves for patients with p53 wildtype (solid line) or mutant (dashed line) tumors using gene expression-based p53 functional category or mutation status. Overall survival analysis comparing the two geneexpression based dendrogram groups (tumors in Figure 5A vs. tumors in Figure 5B) yielded a highly significant difference in survival, which compares favorably with mutation status. Both analyses used 66 tumor samples and included 26 events. Survival analysis on a separate data set (Chang et al., 2005) including 337 tumors and 79 events (C) also yielded highly significant differences based on gene expression classification. 
the signature is indicative of p53 across a wide range of cell backgrounds. If the signature were merely correlated with proliferation, ER status, or another tumor characteristic, then poor concordance with p53 mutational status would be expected in cross validation. The samples where gene expression and mutation status disagree may represent true differences in the functional p53 pathway. For example, the tumor BC606 was p53 wildtype by sequence but clustered with p53 mutants using the 52 gene classifier. This tumor overexpressed MDM2 mRNA (data not shown), a key negative regulator of p53. Among the false negatives (sequence mutant but wildtype expression signature), our analysis of the Miller et al. data showed that misclassified mutants had higher proportions of mutation types that are likely to be less deleterious (missense mutations and in-frame insertion/deletions).

In addition to identifying a stereotyped signature associated with p53 loss, these results demonstrate that the relative importance of p53-regulated functions such as cell cycle control, DNA repair, and apoptosis are subject to significant inter-individual variation. Each cell line displayed a unique p53 response signature. However, similarities according to cell type were also evident. Both of the HMEC-derived cell lines showed a greater response to p53 loss in the untreated state, while the MCF-7 and ZR-75-1 lines showed a stronger p53-regulated signature following DOX treatment. These results extend previous observations [5] suggesting a difference in p53 signaling pathways between luminal and basal-like breast cancers. These inherent differences in p53 signaling could lead to different selection pressure for $\mathrm{p} 53$ loss in each cell type. Such differences could also explain the divergent rates of p53 mutation by subtype that have been reported here and in a population-based study [3].

Our data reconfirmed the complex relation between chemosensitivity and p53 status [22]. Previous reports have demonstrated either heightened chemosensitivity of p53 mutants $[23,24]$ or heightened chemoresistance [25]. This paradox is reflected in our study where the four cell lines we studied varied widely in their DOX sensitivity following p53-knockdown. Because p53 regulates many different pathways, including DNA repair, apoptosis, and cell proliferation, and the balance of these various pathways determines chemosensitivity, it is not surprising to find that both individuals and cell lines have responses to chemotherapy that are difficult to predict. DOX also has many p53-independent toxicity mechanisms, so a divergence in sensitivity across lines may also reflect differences in how DOX toxicity is manifest across lines. These analyses have demonstrated that breast cell lines have individual, distinct responses to p53 loss. The genetic background of a given cell line, including cell type of origin, plays a prominent role in mediating p53 signaling.
A strength of our study was the use of cell line experiments to control a range of variables that influence p53response. The in vitro setting allowed for control of expression of p53 protein, breast cancer subtype, and p53inducing events [26-29]. However, the in vitro approach is limited in that a small number of cell lines can be reasonably examined, representing only a handful of tumors. By combining the in vitro expression data with data from human tumors assayed before and after DOX treatment, we examined a much wider range of individual responses to p53 loss than cell line experiments could reasonably examine, and performed a controlled experiment that cannot be accomplished in humans. Previous studies have characterized p53-responses in breast cancer using gene expression data from tumors and statistical models to try to control the effects of breast cancer heterogeneity. For example, in Miller et al. [14], proliferation and ER status were treated as statistical confounders of the p53-gene expression relation (based on p53 status and outcome both having crude associations with grade and ER status). Thus, the final p53-mutant like gene expression profile presented by Miller et al. [14] was derived using a statistical model that adjusted for these variables. Such adjustment assumes that grade and ER status are causally upstream of p53 status. If grade and ER status are downstream of p53 status, this approach will introduce a bias toward exclusion of grade and ER-associated genes, even though those genes are influenced by p53 loss. In short, the validity of statistical adjustment depends upon having the correct model for the relation between breast cancer subtype, ER status, proliferation and p53 biology. In the presence of heterogeneity, experimental and biologicallybased methods for assessing gene expression in relation to p53 status are preferable to statistical methods.

Many of the genes associated with p53 loss in this analysis were of prior interest in breast cancer. For example, GATA3 is involved in growth control and maintenance of the differentiated state in breast epithelial cells and has been hypothesized to play a role in tumorigenesis of ERpositive breast tumors [30]. p21 (Cip1), CDC2, and CDC25C are genes involved in p53-mediated regulation of cell cycle arrest [31]. Pituitary tumor-transforming 1 is a recently identified oncogene with p53-dependent and p53-independent functions [32]. Thus, as might be expected, many of the direct and indirect targets of p53 identified here are known p53- and cancer-associated genes. Further investigation of the specific p53 targets that are regulated in common across breast cancers and investigation of those that are differentially regulated across breast cancer subtypes will add to our understanding of the biology of breast cancer and breast cancer subtypes. 


\section{Conclusion}

In the presence of breast cancer heterogeneity, controlled experiments in vitro combined with in vivo analyses, allowed for refinement of a p53-associated gene set. The refined 52-gene list excluded genes that were associated with breast cancer subtype and not downstream of p53. This work identified a signature for p53 loss that is shared across breast cancer subtypes and that provided prognostic information and a biologically-relevant gene set.

\section{Competing interests}

The author(s) declare that they have no competing interests.

\section{Authors' contributions}

MAT participated in study design, created the isogenic cell line pairs, performed toxicity assays, western blots, and microarrays, performed analysis and interpretation, drafted and revised the manuscript. JIH participated in study design, creation of cell line pairs, interpretation of results, and manuscript revisions. DSO performed survival and gene ontology analyses. XH performed microarrays. KAH participated in toxicity assays and manuscript revisions. CSB participated in interpretation and critical review of the manuscript. CP participated in study design and coordination, supervision of experimental conduct and analysis, interpretation of results, drafting and revision of the manuscript, and approved the final version. All authors have read and approved the final manuscript.

\section{Additional material}

\section{Additional file 1}

Microsoft Excel spreadsheet (196 kB) containing lists of genes for which expression levels were significantly associated with p53 loss (in 2-class SAM analyses).

Click here for file

[http://www.biomedcentral.com/content/supplementary/14712407-6-276-S1.xls]

\section{Additional file 2}

Cluster figure showing parents and RNAi-transductants for all four parent cell lines (MCF-7, ZR-75-1, HME-CC, and ME16C). Genes included are the 696 genes identified as significant by SAM analyses comparing parents to RNAi-transductants.

Click here for file

[http://www.biomedcentral.com/content/supplementary/14712407-6-276-S2.pdf]

\section{Acknowledgements}

We are grateful to D. Joseph Jerry, Mary Hagen, and William K. Kaufmann for thoughtful review and helpful comments in preparation of this manuscript. This work was supported by funds from the NCI Breast SPORE program to UNC-CH (P50-CA58223-09A I), by NIEHS (UI 9-ESI I39I-03), by $\mathrm{NCl}$ (ROI-CA-I0I227-0I) and by funds from the Breast Cancer Research
Foundation. M.A.T. was supported by NIEHS Individual National Research Service Award (NRSA) 5F32ESOI 2374 and the UNC Lineberger Cancer Control Education Program (R25 CA57726).

\section{References}

I. Borresen-Dale AL: TP53 and breast cancer. Hum Mutat 2003, 21:292-300.

2. Sørlie T, Perou CM, Tibshirani R, Aas T, Geisler S, Johnsen H, Hastie T, Eisen MB, van de Rijn M, Jeffrey SS, Thorsen T, Quist H, Matese JC, Brown PO, Botstein D, Eystein Lonning P, Borresen-Dale AL: Gene expression patterns of breast carcinomas distinguish tumor subclasses with clinical implications. Proc Natl Acad Sci U S A 200I, 98:10869-10874.

3. Carey LA, Perou CM, Livasy CA, Dressler LG, Cowan D, Conway K, Karaca G, Troester MA, Tse CK, Edmiston S, Deming SL, Geradts ], Cheang MC, Nielsen TO, Moorman PG, Earp HS, Millikan RC: Race, breast cancer subtypes, and survival in the Carolina Breast Cancer Study. Jama 2006, 295:2492-2502.

4. Tsutsui S, Ohno S, Murakami S, Hachitanda Y, Oda S: DNA aneuploidy in relation to the combination of analysis of estrogen receptor, progesterone receptor, $\mathrm{p} 53$ protein and epidermal growth factor receptor in $\mathbf{4 9 8}$ breast cancers. Oncology 2002, 63:48-55.

5. Troester MA, Hoadley KA, Sorlie T, Herbert BS, Borresen-Dale AL, Lonning PE, Shay JW, Kaufmann WK, Perou CM: Cell-type-specific responses to chemotherapeutics in breast cancer. Cancer Res 2004, 64:4218-4226.

6. Brummelkamp TR, Bernards R, Agami R: A system for stable expression of short interfering RNAs in mammalian cells. Science 2002, 296:550-553.

7. Tusher V, Tibshirani R, Chu G: Significance analysis of microarrays applied to the ionizing radiation response. Proc Natl Acad Sci U SA 200I, 98:5 I I6-5I II.

8. Eisen MB, Spellman PT, Brown PO, Botstein D: Cluster analysis and display of genome-wide expression patterns. Proc Natl Acad Sci U S A 1998, 95: I 4863-14868.

9. Eisen MB, Brown PO: DNA arrays for analysis of gene expression. Methods Enzymol 1999, 303:179-205.

10. Hosack DA, Dennis G Jr., Sherman BT, Lane HC, Lempicki RA: Identifying biological themes within lists of genes with EASE. Genome Biol 2003, 4:R70.

1I. Perou CM, Sorlie T, Eisen MB, van de Rijn M, Jeffrey SS, Rees CA, Pollack JR, Ross DT, Johnsen H, Akslen LA, Fluge O, Pergamenschikov A, Williams C, Zhu SX, Lonning PE, Borresen-Dale AL, Brown PO, Botstein D: Molecular portraits of human breast tumours. Nature 2000, 406:747-752.

12. Sorlie T, Tibshirani R, Parker J, Hastie T, Marron JS, Nobel A, Deng S, Johnsen H, Pesich R, Geisler S, Demeter J, Perou CM, Lonning PE, Brown PO, Borresen-Dale AL, Botstein D: Repeated observation of breast tumor subtypes in independent gene expression data sets. Proc Natl Acad Sci U S A 2003, 100:8418-8423.

13. Chang HY, Nuyten DS, Sneddon JB, Hastie T, Tibshirani R, Sorlie T, Dai H, He YD, van't Veer LJ, Bartelink H, van de Rijn M, Brown PO, van de Vijver M]: Robustness, scalability, and integration of a wound-response gene expression signature in predicting breast cancer survival. Proc Natl Acad Sci U S A 2005, 102:3738-3743.

14. Miller LD, Smeds J, George J, Vega VB, Vergara L, Ploner A, Pawitan $Y$, Hall P, Klaar S, Liu ET, Bergh J: An expression signature for $\mathrm{p} 53$ status in human breast cancer predicts mutation status, transcriptional effects, and patient survival. Proc Natl Acad Sci U S A 2005, I 02: I3550- I3555.

15. Benito M, Parker J, Du Q, Wu J, Xiang D, Perou CM, Marron JS: Adjustment of systematic microarray data biases. Bioinformatics 2004, 20:105-I|14.

16. van 't Veer LJ, Dai $H$, van de Vijver MJ, He YD, Hart AA, Mao M, Peterse HL, van der Kooy K, Marton MJ, Witteveen AT, Schreiber G], Kerkhoven RM, Roberts C, Linsley PS, Bernards R, Friend SH: Gene expression profiling predicts clinical outcome of breast cancer. Nature 2002, 415:530-536.

17. Rhodes DR, Yu J, Shanker K, Deshpande N, Varambally R, Ghosh D, Barrette T, Pandey A, Chinnaiyan AM: Large-scale meta-analysis of cancer microarray data identifies common transcriptional profiles of neoplastic transformation and progression. Proc Natl Acad Sci U S A 2004, 10 I:9309-9314. 
18. Lamb J, Ramaswamy S, Ford HL, Contreras B, Martinez RV, Kittrell FS, Zahnow CA, Patterson N, Golub TR, Ewen ME: A mechanism of cyclin DI action encoded in the patterns of gene expression in human cancer. Cell 2003, I I 4:323-334.

19. Bild AH, Yao G, Chang JT, Wang Q, Potti A, Chasse D, Joshi MB, Harpole D, Lancaster JM, Berchuck A, Olson JA Jr., Marks JR, Dressman HK, West M, Nevins JR: Oncogenic pathway signatures in human cancers as a guide to targeted therapies. Nature 2006, 439:353-357.

20. Pharoah PD, Day NE, Caldas C: Somatic mutations in the p53 gene and prognosis in breast cancer: a meta-analysis. $\mathrm{Br} J \mathrm{Can}-$ cer 1999, 80:1968-1973.

21. Soussi T, Asselain B, Hamroun D, Kato S, Ishioka C, Claustres M, Beroud C: Meta-analysis of the p53 mutation database for mutant p53 biological activity reveals a methodologic bias in mutation detection. Clin Cancer Res 2006, 12:62-69.

22. Ferreira CG, Tolis C, Giaccone G: p53 and chemosensitivity. Ann Oncol 1999, 10:1011-1021.

23. Fan S, el-Deiry WS, Bae I, Freeman J, Jondle D, Bhatia K, Fornace AJ Jr., Magrath I, Kohn KW, O'Connor PM: p53 gene mutations are associated with decreased sensitivity of human lymphoma cells to DNA damaging agents. Cancer Res 1994, 54:5824-5830.

24. Fan S, Smith ML, Rivet DJ 2nd, Duba D, Zhan Q, Kohn KW, Fornace AJ Jr., O'Connor PM: Disruption of p53 function sensitizes breast cancer MCF-7 cells to cisplatin and pentoxifylline. Cancer Res 1995, 55:1649-1654.

25. Brachman DG, Beckett M, Graves D, Haraf D, Vokes E, Weichselbaum RR: p53 mutation does not correlate with radiosensitivity in 24 head and neck cancer cell lines. Cancer Res 1993, 53:3667-3669.

26. Maxwell SA, Davis GE: Differential gene expression in p53mediated apoptosis-resistant vs. apoptosis-sensitive tumor cell lines. Proc Natl Acad Sci U S A 2000, 97: I3009-130I4.

27. Zhao R, Gish K, Murphy M, Yin Y, Notterman D, Hoffman WH, Tom E, Mack DH, Levine AJ: Analysis of p53-regulated gene expression patterns using oligonucleotide arrays. Genes Dev 2000, I 4:98|-993.

28. Madden SL, Galella EA, Zhu J, Bertelsen AH, Beaudry GA: SAGE transcript profiles for p53-dependent growth regulation. Oncogene 1997, I 5: 1079-1085.

29. Polyak K, Xia Y, Zweier JL, Kinzler KW, Vogelstein B: A model for p53-induced apoptosis. Nature 1997, 389:300-305.

30. Usary J, Llaca V, Karaca G, Presswala S, Karaca M, He X, Langerod A, Karesen R, Oh DS, Dressler LG, Lonning PE, Strausberg RL, Chanock S, Borresen-Dale AL, Perou CM: Mutation of GATA3 in human breast tumors. Oncogene 2004, 23:7669-7678.

31. Harris SL, Levine AJ: The p53 pathway: positive and negative feedback loops. Oncogene 2005, 24:2899-2908.

32. Hamid T, Kakar SS: PTTG and cancer. Histol Histopathol 2003, 18:245-25I.

\section{Pre-publication history}

The pre-publication history for this paper can be accessed here:

http://www.biomedcentral.com/1471-2407/6/276/pre pub

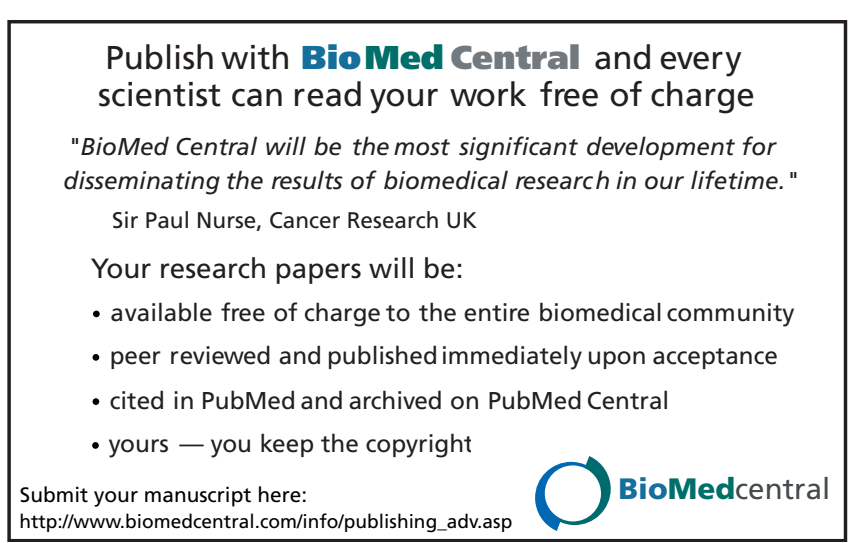

